\title{
Evaluation of the Same Day Diagnosis of TB Microscopy in Comparison with Spot-Morning-Spot Method in Selected Public Health Institutions, Addis Ababa, Ethiopia
}

\author{
Shemsu Kedir Juhar ${ }^{*}$, Sisay Kebede Gebregeorgis ${ }^{1}$, Awad Mohammed Amdalla ${ }^{1}$, Yemsirach Reta Silishe ${ }^{1}$, \\ Mulualem Agonafir ${ }^{1}$, Kassu Desta ${ }^{2}$ \\ ${ }^{1}$ Ethiopian Public Health Institute, Ethiopia; ${ }^{2}$ College of Health Sciences Department of Medical Laboratory Sciences, \\ Addis Ababa University, Ethiopia
}

\begin{abstract}
Background: The need to collect serial sputum specimens over multiple patient visits for pulmonary tuberculosis results in a protected diagnostic process with rates of patients with high rates of patient dropout. Recent studies on Spot Morning Spot (SMS) method of examination PTB reported that the first two specimens have high smear positivity in line with this WHO changed its policy to minimize the number of sputum specimens from three to two.

Methods and Materials: Across-sectional study was conducted in 16 conveniently selected private clinics, governmental health centers, public and private hospitals from September 2017-December 2018. Individuals attending the selected health institutions for the diagnosis of MTB submitted three sputum samples for routine diagnosis (the standard approach). One additional sample was collected $1 \mathrm{~h}$ after the first sputum (the same-day approach). One sputum sample was cultured. The diagnosis was performed using ZN sputum smear microscopy and Light-Emitting Diodes Fluorescent Microscopy (LED-FM) technique. Data were entered and analyzed using SPSS version 16 . We used sensitivity, specificity and predictive values for the different methods.

Result: A total of 209 participants enrolled, 43 (21\%) were identified culture positive, 39 (18.7\%) were detected by the same day approach and 40 (19.1\%) by the standard approach. On the other hand, LED-FM and ZN microscopy detected 39 (18.1\%) and 48 (23\%) tuberculosis cases respectively. Sensitivity was $88.4 \%$ for ZN microscopy and 95.3\% for LED-FM and the specificity was $99.4 \%$ and $95.9 \%$ for ZN and LED-FM microscopy respectively.

Conclusion: Using the same day approach together with LED-FM would reduce workload, TAT, patient drop out and increase the smear detection rate. Therefore it is necessary to give in-service and off service training for health personnel towards the use of LED-FM, conventional approach and the same day approach in the diagnosis of TB.

Keywords: Mycobacterium tuberculosis; antibiotics; Smear; Non-tuberculosis mycobacterium
\end{abstract}

\section{INTRODUCTION}

Tuberculosis is a curable and preventable disease. It is an infectious bacterial disease caused by Mycobacterium tubercle, an Acid Fast Bacilli as Koch stated and it is spread by aerosolization of droplet nuclei bearing Mycobacterium tuberculosis particles released from the lung of a patient with the cavitary pulmonary or laryngeal disease. According to WHO TB is the second most common cause of deaths due to infectious disease, and current trends suggest that TB will still be among the 10 leading causes of global disease burden in the year 2020 $[1,2]$.

In developing countries, diagnosis of Pulmonary Tuberculosis (PTB) depends primarily on the identification of Acid-Fast Bacilli (AFB) using Ziehl-Neelsen sputum smear microscopy, a

Corresponding author: Shemsu Kedir Juhar, Ethiopian Public Health Institute, Ethiopia, Tel: +251913768756; E-mail: shemsukedir1@gmail.com

Received date: May 27, 2019; Accepted date: July 11, 2019; Published date: July 20, 2019

Citation: Juhar SK, Gebregeorgis SK, Amdalla AM, Silishe YR, Agonafir M, Desta K (2019) Evaluation of the Same Day Diagnosis of TB Microscopy in Comparison with Spot-Morning-Spot Method in Selected Public Health Institutions, Addis Ababa, Ethiopia. J Trop Dis 7:323.

Copyright: (C) 2019 Juhar SK, et al. This is an open-access article distributed under the terms of the Creative Commons Attribution License, which permits unrestricted use, distribution and reproduction in any medium, provided the original author and source are credited. 
technique more than 100 years old. The sensitivity of this method varies and depends upon the collection of sufficient sputum, proper preparation of smears, good staining technique, careful examination of smears, and availability of a good microscope. Several methods have been tried to improve smear microscopy for AFB [3]. On the other hand, Light Emitting Diode (LED) microscopes are less expensive, require less power and are able to run on batteries, the bulbs have a very long halflife. These qualities make LED microscopy feasible for use in resource-limited settings, having the potential to bring the benefits of fluorescent microscopy (improved sensitivity and efficiency) where needed most [4].

Recent studies examining the yield of serial sputum specimens, usually collected as spot-morning-spot, have reported that the majority of patients with smear-positive PTB are identified by the first two sputum specimens and the World Health Organization (WHO) has recently changed its policy in this respect, reducing the minimum number of sputum specimens examined for each patient from three to two [5].

The diagnostic accuracy of conventional case-finding strategies compared to an approach where two consecutive sputum specimens ('spot-spot') are examined on the same day (so-called 'front-loaded' or 'same-day-diagnosis'), and also assessed whether patient drop-out from the diagnostic pathway can be reduced as a result [6].

\section{MATERIALS AND METHODS}

\section{Study design and period}

A cross-sectional study was conducted from September 2017 to December 2018 in the selected 16 governmental, private and uninformed health hospitals. A study site was selected from those health facilities that have both $\mathrm{TB}$ clinics, run direct microscopy for AFB regularly and participate in EQA, from those study sites will be selected conveniently from the region. In addition, patients who have visited those health facilities, and health personals that are working in those facilities, voluntary and give their consent to participate were included in the study.

A sample size of the study population was all patients who give sputum sample for TB microscopy included conveniently from all sites of the study setting within the study period.

N.B: the study is method comparison, the maximum sample size in method comparison is from 100-200 because of this we select a total of 220 samples with its non-response rate for this study.

\section{Statistical analysis}

Data were double entered using EPI-INFO version 3.5.1 and further statistical analyses were made in SPSS version 16. ChiSquare was used to assess the associations of different variables with methods, health personals KAP and health personals Knowledge with the same day diagnosis of $\mathrm{TB}$ microscopy. $\mathrm{p}<0.05$ was statistically significant.

The sensitivity, specificity, positive and negative predictive values of the sputum smear examinations including their $95 \%$ Confidence Intervals (CI) were calculated by using the sputum culture results as the "gold standard." p<0.05 was considered being statistically significant.

\section{Sample collection for microscopy diagnosis}

A sputum sample was collected from patients who were come for AFB diagnosis in those health facilities. During collection four consecutive sputum sample at least $5-10 \mathrm{ml}$ sputum samples were collected in clean, sterile, leak-proof, screw caped widemouth $(35 \mathrm{~mm}$ in diameter and volume capacity of $28-50 \mathrm{ml}$ ), one use disposable containers with easily labeled wall mark including date of collection, laboratory serial number, age, and sex after careful instruction is given to patients [7].

\section{Laboratory methods}

Spot and after one hour x-spot sample was collected on the first day and morning and spot sample was collected on the second day. From this sample, the smear was prepared for $\mathrm{ZN}$ microscopy and LED_FM technique and stained using AFB reagent and Auramine $O$ phenol method reagent respectively. All positive and negative samples were transported to the Ethiopian Public Health Institute (EPHI) TB laboratory to confirm the result using LJ medium and biochemical taste.

\section{Direct smear preparation and staining}

From each spot and X-spot sample of three smears for reproducibility check and from a total of four sample from each one smear of two sets of direct smears were prepared by taking a small portion of the purulent part of the sputum with an applicator stick, and smearing it on a microscope slide, which will then dried in the air and fumed on a hot plate. One set was stained with $\mathrm{ZN}$ and the other set was stain with the auramine o phenol method [7].

\section{Inoculation and incubation of culture media}

Primarily condensed moisture observed at the bottom of culture medium slants were removed before inoculation. Inoculation for primary isolation, identification, and susceptibility testing of Mycobacterium was done at bio-safety level II cabinet, using pipettes each slope was inoculated $150 \mu$ of the centrifuged sediment, distributed over the surface. Two slopes of LJ medium were inoculated per specimen. But the preparation of inoculums for susceptibility testing was according to the McFarland standards of inoculums preparation procedure stated in the annex part [8].

All cultures were incubated at $35^{\circ} \mathrm{C}-37^{\circ} \mathrm{C}$ until growth is observed and cultures were discarded as negative after eight and six weeks for primary isolation and susceptibility testing respectively. Inoculated media was preferably be incubated in a slanted position for at least 24 hours to ensure even distribution of inoculums. Thereafter, if incubator space is needed, bottles were placed upright. Caps were tightened to minimize evaporation and drying of media [8]. 


\section{RESULTS}

A total of 220 patients with suspected pulmonary tuberculosis were enrolled from September 2017 to December 2018 in three health centers, two private clinics and 11 hospitals which are participating in EQA. Socio-demographic characteristics such as age and sex were obtained for all study subjects and analyzed (Table 1). The mean age was 39 years with a range of 13-78. 148 $(70.8 \%)$ were males. with a male to female ratio of 2.1 : 1 (Table $1)$.

Table 1: Distribution of Socio-demographic characteristics of patients by age and sex $(n=220)$, September 2017-December 2018 Addis Ababa, Ethiopia.

\begin{tabular}{lll}
\hline Variables & Frequency & Percent $(\%)$ \\
\hline Age group & & \\
\hline $10-20$ & 7 & 3.3 \\
\hline $21-30$ & 37 & 17.7 \\
\hline $31-40$ & 89 & 44.6 \\
\hline $41-50$ & 46 & 20 \\
\hline$\geq 51$ & 31 & 14.8 \\
\hline Sex & 148 & 70.8 \\
\hline Male & 61 & 29.2 \\
\hline Female & & \\
\hline
\end{tabular}

\section{Results of SS, SMS, SSM and SM approach by ZN staining using culture as the gold standard}

A total of 220 pulmonary tuberculosis suspected patients were included in the study. Among them, 11 patients did not fulfill the inclusion criteria and were excluded. From these 209 patients, a total of 836 sputum specimens were collected. Four sputum samples from each patient were collected for spot, extraspot, morning and second-day spot sample. SMS samples were analyzed by each health facility laboratory; spot and extra-spot sample analyzed by the principal investigator.

Out of a total of 209 cases, it was observed that 43 (20.6\%) cases were diagnosed as pulmonary tuberculosis by culture method as a gold standard. SMS and SM showed the nearest result as 40 (19.1\%) positive cases, in which 1 case was found as false positive. On the other hand, SS and SSM detected 39 (18.7\%) and $40(19.1 \%)$ cases positive, respectively (Figure 1). Both missed 1 case each. The difference in the case-yields between SS with SMS, SSM and SM approaches were $0.4 \%, 0.9 \%$, and $0.45 \%$, respectively the case detection of each approach was almost found to be similar.

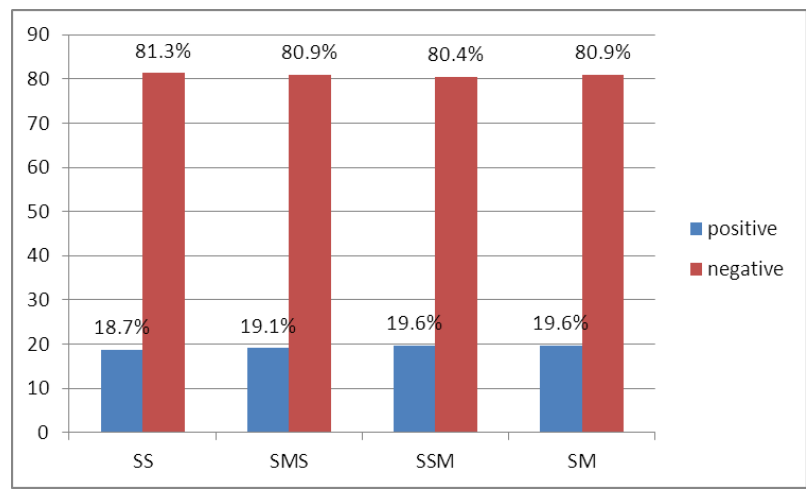

Figure 1: Distribution of microscopy TB positive rate using different specimen collection approach SS, SMS, SSM and SM approach by ZN staining. (SS: Spot And Extra-Spot; SMS: Spot-Morning-Spot; SSM: Spot-Extra-Spot-Morning; SM: Spot-Morning).

\section{Comparison of the same day (spot, extra-spot) and spot- morning-spot (SMS) sampling method using ZN}

Morning sputum specimens of 209 (100\%) of the patients enrolled were cultured, and 43 were found to be culture-positive. The analysis was conducted on 209 patient's sputum sample using $\mathrm{ZN}$ as shown in (Table 2). Out of the 43 culture positives, first spot and extra-Spot (SS) and Spot-Morning-Spot (SM) identified $38(88.4 \%)$ and 39 (90\%) smear positives, respectively. Two percent of the cases were recognized as false positive by both approaches. Both SS and SMS approach missed 11.6\% and 9.3\% of the cases, respectively. All 165 culture-negative samples were found to be smearing negative by both approaches. The sensitivity, specificity, PPV, NPV and accuracy using culture as a gold standard were $88.4 \%, 99.4 \%, 97 \%, 97.3 \%, 97.1 \%$ for SS, while 90.4\%, 99.4\%, 98\%, 97.5\%, 97.6\% were for SMS approaches, respectively (Table 2 ).

This study indicates that the sensitivity and specificity of Spot, extra-Spot (SS) were non-inferior to the sensitivity and specificity of spot-morning-spot (SMS) approach.

Table 2: Comparison of the spot, extra-spot (one-day sampling) and spot, morning and spot (two days sampling) sampling method by ZN staining method using culture as a gold standard September 2017-December 2018 Addis Ababa, Ethiopia.

Culture (n=209)

\begin{tabular}{llllllll}
\hline Approach & Positive & Negative & Sensitivity & Specificity & Accuracy & PPV $^{*}$ & NPV $^{£}$ \\
\hline Same day (SS & & & & & & \\
\hline Positive & 38 & 1 & 88.4 & 99.4 & 97 & 97.3 & 97.1 \\
\hline
\end{tabular}




\begin{tabular}{|c|c|c|c|c|c|c|c|}
\hline Negative & 5 & 165 & & & & & \\
\hline \multicolumn{8}{|c|}{ Standard $\left(\mathrm{SMS}^{\diamond}\right)$} \\
\hline Positive & 39 & 1 & 90.4 & 99.4 & 98 & 97.5 & 97.6 \\
\hline Negative & 4 & 165 & & & & & \\
\hline
\end{tabular}

\section{Evaluation of LED-fluorescence microscopy and ZN smears microscopy based on the same day diagnosis using spot and extra-spot samples}

A total of 209 pulmonary tuberculosis suspected patients participated in the study. Out of the study subjects, a total of 418 spot and extra-spot sputum specimens were collected for our study. Morning sputum specimens of 209 (100\%) of the patients enrolled were cultured, and 43 were culture-positive. The analysis was conducted on 209 patient's spot, extra-spot sputum sample (same day diagnosis) using ZN stain bright field microscopy and AO stained LED-FM as shown in (Table 3). First spot and extra-Spot (the same day sampling) 39 and 48 were identified positively by $\mathrm{ZN}$ bright field microscopy and $\mathrm{AO}$ stained LED-FM respectively, of 418 sputum sample. From a total of 43 culture positive patients, $88.4 \%$ were identified as positive, $2 \%$ were falsely positive and $11.6 \%$ were missed by $\mathrm{ZN}$ bright field microscopy. By AO LED-FM, 95.3\% were identified positive, $16.2 \%$ were falsely positive and $5 \%$ were missed. The sensitivity, specificity, PPV, NPV, and accuracy yielded with LED-fluorescent microscopy using culture as a gold standard were $95.3 \%$, 95.9\%, 85.4\%, 98.8\%, and 95.7\%, respectively. While for $\mathrm{ZN}$ stained bright field microscopy were $88.4 \%$, 99.4\%, 97.3\%, 97.1\%, and 97\%, respectively (Table 3).

Table 3: Evaluation of LED-fluorescence microscopy in comparison to ZN smear microscopy based on the same day diagnosis (spot, extra-spot) sample using culture as a golden standard ( $\mathrm{n}=209)$, September 2017-December 2018 Addis Ababa, Ethiopia.

Method Culture $\quad$ Sensitivity $\quad$ Specificity $\quad \mathrm{PV}^{\star} \quad \mathrm{NPV}^{\bullet} \quad$ Accuracy

\begin{tabular}{|c|c|c|c|c|c|c|c|}
\hline & Positive & Negative & & & & & \\
\hline \multicolumn{8}{|c|}{$\mathrm{ZN}$ stained $\left(\mathrm{BFM}^{\diamond}\right)$} \\
\hline positive & 38 & 1 & $88.40 \%$ & $99.40 \%$ & $97.30 \%$ & $97.10 \%$ & $97 \%$ \\
\hline Negative & 5 & 165 & & & & & \\
\hline
\end{tabular}

AO stained (LED-FM*)

\begin{tabular}{|c|c|c|c|c|c|c|c|}
\hline positive & 41 & 7 & $95.30 \%$ & $95.90 \%$ & $85.40 \%$ & $98.80 \%$ & $95.70 \%$ \\
\hline Negative & 2 & 159 & & & & & \\
\hline
\end{tabular}

$\diamond$ : Bright filed microscopy; $\triangleleft$ Positive predictive value; $\bullet$ : Negative predictive value; $*$ : Light emitting diode fluorescent microscopy

The precision of $\mathrm{ZN}$ smears microscopy in the same-day diagnosis using culture as the golden standard

From 209 suspected patients, a total of 836 spot and x-spot sputum specimens were collected. From each sample, three smears were prepared and stained by $\mathrm{ZN}$ staining method to check the reproducibility of the $\mathrm{ZN}$ staining method based on spot and $\mathrm{x}$-spot sampling method. The smears were examined by three different qualified individuals.

From the first, second and third smear of spot sputum samples, a total of 36,35 and 36 were smearing positive, respectively. In the same analysis from the $x$-spot samples, a total of 35,36 , and
39 smears were found to be positives, respectively, out of the 43 culture positive samples. The reproducibility (precision) measurements of the spot and x-spot sample smear microscopy were analyzed. The average mean was 0.18 and 18.6 the average standard error 0.046 and 0.043 , range of standard error 0.045-0.049 and 0.036-0.047 respectively. The mean CV were $0.27(27 \%)$ and $0.15(15 \%)$, the range $0.149-0.158$ and 0.146-0.155 respectively (Table 4).

The reproducibility (precision) of results was good which was indicated with a good inter-reader agreement between the laboratory personnel was observed with the average kappa value 
0.723 and 0.873 with the range of $0.846-0.862$ and $0.846-0.911$, respectively.

Table 4: Precision of ZN smears microscopy in the same-day diagnosis, in Selected Health Facility, September 2017-December 2018 Addis Ababa, Ethiopia.

\begin{tabular}{|c|c|c|c|c|c|c|c|c|c|c|c|}
\hline \multirow{2}{*}{$\begin{array}{l}\text { ZN smear } \\
\text { Spot sample }(n=209)\end{array}$} & & \multicolumn{2}{|c|}{ culture } & \multirow{2}{*}{$\begin{array}{l}\text { Negative } \\
\mathrm{Nq}(\%) \diamond\end{array}$} & \multirow{2}{*}{$\begin{array}{l}\text { Positive } \\
\text { N (\%) }\end{array}$} & \multicolumn{3}{|c|}{ smear grading } & \multirow[t]{2}{*}{$\mathrm{SD}^{*}$} & \multirow[t]{2}{*}{ CVA } & \multirow[t]{2}{*}{ Kapp a.v } \\
\hline & & -ve $\bullet$ & $+v e \triangle$ & & & scanty & $1+$ & $2+$ & & & \\
\hline \multirow[t]{2}{*}{$1^{\text {st }}$ slide } & + ve & 2 & 36 & $171(81.8)$ & $38(18.2)$ & 14 & 20 & 4 & 0.387 & 0.149 & 0.862 \\
\hline & $-\mathrm{ve}$ & 164 & 7 & & & & & & & & \\
\hline Total & & 166 & 43 & & & & & & & & \\
\hline \multirow[t]{2}{*}{$2^{\text {nd }}$ slide } & $+\mathrm{ve}$ & 2 & 35 & $172(82.3)$ & $37(17.2)$ & 14 & 20 & 3 & 0.383 & 0.146 & 0.846 \\
\hline & $-v e$ & 164 & 8 & & & & & & & & \\
\hline Total & & 166 & 43 & & & & & & & & \\
\hline \multirow[t]{2}{*}{$3^{\text {rd }}$ slide } & $+\mathrm{ve}$ & 3 & 35 & $170(81.3)$ & 39 (18.7) & 14 & 20 & 4 & 0.391 & 0.158 & 0.848 \\
\hline & $-\mathrm{ve}$ & 163 & 7 & & & & & & & & \\
\hline Total & & 166 & 43 & & & & & & & & \\
\hline
\end{tabular}

X-Spot sample $(n=209)$

\begin{tabular}{|c|c|c|c|c|c|c|c|c|c|c|c|}
\hline \multirow[t]{2}{*}{$1^{\text {st }}$ slide } & $+v e$ & 2 & 35 & $172(82.3)$ & $37(17.7)$ & 14 & 19 & 4 & 0.383 & 0.146 & 0.846 \\
\hline & -ve & 164 & 8 & & & & & & & & \\
\hline Total & & 166 & 43 & & & & & & & & \\
\hline \multirow[t]{2}{*}{$2^{\text {nd }}$ slide } & $+\mathrm{ve}$ & 2 & 36 & $171(81.8)$ & 38 (18.2) & 14 & 20 & 4 & 0.387 & 0.149 & 0.862 \\
\hline & -ve & 164 & 7 & & & & & & & & \\
\hline Total & & 166 & 43 & & & & & & & & \\
\hline \multirow[t]{2}{*}{$3^{\text {rd }}$ slide } & $+\mathrm{ve}$ & 2 & 39 & $168(80.4)$ & $41(19.6)$ & 16 & 21 & 4 & 0.393 & 0.155 & 0.911 \\
\hline & -ve & 162 & 4 & & & & & & & & \\
\hline Total & & 166 & 43 & & & & & & & & \\
\hline
\end{tabular}

$\bullet:$ Negative; $\triangle$ : Positive; $\theta$ : Number; $\diamond:$ Present; $*$ : Standard devotions;

Coefficient variation,

\section{Quality control}

Positive and Negative control specimens and smears were used in every batch of culture and staining. Blinded reading of the slides was done by another group of four independent laboratory technologists. An experienced technician independent from the two groups, read an arbitrary $10 \%$ positive and $5 \%$ negatives slide that are selected randomly. In addition to this, the same technician re-read all the smears with discrepant results. All reagents were prepared in accordance with the Standard Operating Procedure (SOPs) used at the laboratory.

\section{DISCUSSION}

Direct sputum smear microscopy is the most widely used test for the diagnosis of pulmonary Tuberculosis (TB), available in most primary health care laboratories. The majority of laboratories use conventional light microscopy to examine Ziehl-Neelsen stained direct smears, documented to be highly specific in areas with a high prevalence of $\mathrm{TB}$ but with varying sensitivity 
(20\%-80\%). Besides being labor-intensive, direct sputum smear microscopy may have considerable patient costs and inconvenience associated with the need to submit multiple sputum specimens over a period of up to three days. A number of $\mathrm{TB}$ control programs have reported high rates of initial patient default as a result [9].

This study examined different sputum sample collection approaches comparing different microscopy techniques that are used in the diagnosis of PTB on sputum specimens. Very much comparable positive case yields of spot-morning-spot (19.1\%), spot-morning $(19.1 \%)$, spot-x-spot $(18.7 \%)$ and spot-x-spotmorning $(19.1 \%)$ were found in the current study. The detection capacity of each approach was almost similar to the study conducted in Ethiopia, Nepal, Nigeria, and Yemen by Andy Ramsey et al. [4].

Our study revealed that the difference in missed cases by the same day diagnosis approach and the conventional method was minimal. Furthermore, this study showed that there is no significant difference in smear positivity rate, sensitivity, and specificity between the one-day sampling and two-day conventional sampling methods in $\mathrm{TB}$ diagnosis. There are different studies reported in different parts of the world that corroborate our findings [10-13].

To reduce the high direct and indirect patient costs and inconvenience associated with multiple health facility visits, patient cost and failure to complete smear evaluation, our findings suggest that using a one-day sputum collection (a spot, x-spot sputum specimen) with strong EQA could be sufficient for the diagnosis of PTB. This would reduce workload, improve turnaround time, increase the smear detection rate and reduce transmission of TB.

Also in this study, we have determined the precision (reproducibility) of $\mathrm{ZN}$ staining technics in the same day diagnosis of PTB. It showed that the three smear microscopy result of each spot samples, the x-spot sample smear microscopy, and slide grading were found to be the same in the repeated examination of the smear by different laboratory personals. Also based on the standard devotions and coefficient of variation of the repeated measurements obtained in this study indicate that $\mathrm{ZN}$ technique had high precision in the diagnosis of PTB using the same day diagnosis sampling method. Moreover, our study had shown good inter-reader agreement between the laboratory personnel.

There is no study done on the precision or reproducibility of the same day diagnosis and we were not able to make comparisons.

The backbone of TB diagnosis worldwide continues to be smear microscopy. Thus, increasing the sensitivity of smear microscopy could have a large impact on global TB case detection rates. As a result, there have been several initiatives to optimize smear microscopy including changes in specimen collection procedures, specimen processing, and microscopy techniques [5].

Recent technical developments have the potential to improve some of the shortcomings of the smear diagnosis of TB. These include the development of illumination systems based on LEDs
(Light-Emitting Diodes), which resulted in LED Fluorescence Microscopy (LED-FM) becoming commercially available. There was also an initiative from our country's side to use LED fluorescent microscopes in selected sites which are found nationwide (personal communication).

In the current study on evaluation of the microscopic techniques by comparing them with the gold standard culture technique, it was found that LED-fluorescent microscopy was more sensitive than bright field microscopy. For instance, with ZN stain, 5 (11.6\%) cases were missed and detected as a false negative. On the other hand, auramine stain LED-fluorescent microscopy missed only 2 (4\%) and detected as false negative using culture as the gold standard. The differences of sensitivity, specificity, PPV, NPV and accuracy between ZN and LED fluorescent microscopy were $6.9 \%, 3.5 \%, 11.9 \%, 1.7 \%$, and $1.3 \%$, respectively. These were almost comparable with other several studies $[14,15]$.

The improved efficacy of LED fluorescence microscopy could compliment the reduced sensitivity of the regular bright field microscopy thereby ensuring rapid smear results and effective treatment of the most infections TB cases. It is then imperative to use LED microscopy of sputum by AO staining along with ZN stain for the diagnosis of pulmonary tuberculosis by implementing good quality assurance systems to diagnose more patients and get better treatment outcomes.

\section{CONCLUSION}

This study shows that the time, number of visits, workload, turnaround time and patients' costs to complete smear microscopy could be reduced by one day (same day or frontloading) collection of sputum specimens. Furthermore, LED-based Fluorescence Microscopy (FM) was shown to be more sensitive than $\mathrm{ZN}$ for diagnosis of pulmonary tuberculosis. Hence, LED microscopy of sputum by AO staining can be used effectively along with $\mathrm{ZN}$ stain for the diagnosis of pulmonary tuberculosis, Using this method (LED) in clinical laboratories with the one-day sampling approach could help process large specimen numbers and increase the smear detection rate thereby decreasing false negative smears.

\section{REFERENCES}

1. WHO. Global tuberculosis control report. 2014.

2. Mathema B, Kurepina NE, Bifani PJ, Kreiswirth BN. Molecular epidemiology of tuberculosis: Current insights. Clin Microbiol Review. 2006;19:658-685.

3. Nyagosya R, Andersen A, Vanleth F, Pascal M, Mugomela A, Friis H. Risk factor for smear negative and culture positive results among pulmonary tuberculosis patients in Mwanza, Tanzania. Open Tropical Med J. 2008;1:68-73.

4. Andy R, Yassin MA, Cambanis A, Hirao S, Almotawa A, Gammo $\mathrm{M}$, et al. Front-loading sputum microscopy services: An opportunity to optimize smear-based case detection of tuberculosis in high prevalence countries. J Tropical Med. 2009:6.

5. Selvakumar N, Fathima R, Garg R, Rajasekaran S, Nalini SM, Thyagarajan $\mathrm{K}$, et al. Evaluation of the phenol ammonium sulfate sedimentation smear microscopy method for diagnosis of pulmonary tuberculosis. J Clinl Microbiol. 2002;40:3017-3020. 
6. WHO. Global tuberculosis control report. 2010.

7. Central TB division directorate general of health services ministry of health and family welfare, Nirman Bhavan, manual for sputum smear fluorescence microscopy revised national tuberculosis control program Nirman Bhavan, New Delhi. 2006.

8. BACTEC 960 working manual adapted from BD Diagnostic Systems, Becton, Dickinson and Company. 2001.

9. Ben JM, Wendy B, Katrien P, Anneke C, Hesseling N, Wasserman $\mathrm{E}$, et al. Use of light-emitting diode fluorescence microscopy to detect acid-fast bacilli in sputum. Oxford J Med Clin Infect Dis. 2008;47:203-207.

10. Shenai S, Minion J, Vadwai V, Tipnis T, Shetty S, Salvi A, et al. Evaluation of light emitting diode-based fluorescence microscopy for the detection of mycobacteria in a tuberculosis-endemic region. Int J Tuberculosis Lung Dis. 2017;15:483-488.

11. Somoskovi A, Hotaling JE, Fitzgerald M (2009) Lessons from a proficiency testing event for acid-fast microscopy. Chest. 2009; 120:250-257.
12. WHO. Expert group meeting report. Approaches to improve sputum smear microscopy for tuberculosis diagnosis geneva. 2009.

13. Minion J, Shenai S, Vadwai V, Tipnis T, Greenaway C, D Menzies, et al. Fading of auramine-stained mycobacterial smears and implications for external quality assurance. J Clin Microbiol. 2011;49:2024-2026.

14. Cattamanchi A, Huang L, Worodria W, Boon SD, Kalema N, Katagira W, et al. Integrated strategies to optimize sputum smear microscopy: a prospective observational study. Am J Respir Crit Care Med. 2017;183:547-551.

15. Hirao S, Yassin MA, Khamofu HG, Lawson L, Cambanis A, Ramsay A, et al. Same-day smears in the diagnosis of tuberculosis. Trop Med Int Health. 2007;12:1459-1463. 